\title{
Morphometric evaluation of the sphenopalatine foramen for endonasal surgery*
}

\author{
Adriana B. Antunes Scanavini ${ }^{1}$, J.A.C. Navarro ${ }^{2}$, Silvia R.M.C. Megale ${ }^{1}$, \\ Ronaldo Silveira Lima ${ }^{1}$, Wilma T. Anselmo-Lima ${ }^{3}$ \\ 1 Department of Ophthalmology, Otorhinolaryngology and Head and Neck Surgery, School of Medicine of \\ Ribeirão Preto, University of São Paulo, Ribeirão Preto, SP, Brazil. \\ 2 Department of Anatomy, Dental School of Bauru, Bauru, SP, Brazil. \\ 3 Associated Professor of the Department of Ophthalmology, Otorhinolaryngology and Head and Neck Surgery, \\ School of Medicine of Ribeirão Preto, University of São Paulo, Ribeirão Preto, SP, Brazil.
}

SUMMARY Objective: Ligation of the sphenopalatine artery is used to treat severe nasal haemorrhage. The objective of the study was to investigate the numerical variation of the sphenopalatine foramen (SPF), its relation to the ethmoid bone crista of the palatine bone in the lateral nasal wall, its distance from the anterior nasal spine and the angle between this distance and the anterior nasal floor.

Material and Methods: Fifty-four hemiskulls were submitted to anatomical study and measurements using the Image Tool 3.0 software.

Results: The SPF was single in $87 \%$ of the specimens, and more than one orifice was present in $13 \%$. It was possible to establish a relation with the ethmoid crista, which is a surgical reference for the SPF location. The mean values of the measurements were significantly higher in the hemifaces than in the hemiskulls ranging from 54 to $63 \mathrm{~mm}$, and angulation ranged from 20 to 32 degrees.

Conclusions: In most specimens studied, the SPF was single and located in the superior nasal meatus. The distances measured suggest that these values can be used as distance references for the use of the endoscope for ligation or endonasal cauterization of the branches of the sphenopalatine artery, preventing possible errors and complications.

Key words: sphenopalatine foramen, lateral nasal wall, ethmoid crista, sphenopalatine artery, nasal haemorrhage, arterial ligation.

\section{INTRODUCTION}

Nasal haemorrhage are among the most common problems faced by surgeons in otorhinolaryngology, representing medical emergencies in the more severe cases. In these situations, the efficacy of treatment is directly related to intervention as close as possible to the bleeding site, i.e. to the identification of the most precise topodiagnosis.

The sphenopalatine foramen (SPF) is an incisure in the superior margin of the palatine bone between the orbital and sphenoid processes, which is transformed into a foramen when the palatine bone articulates with the sphenoid bone on the lateral nasal wall. Its position on the lateral nasal wall is related to the ethmoid crista of the palatine bone, where it fixes to the tail of the middle turbinate. The SPF can be located in the middle or superior meatus or in both and is considered to be an anatomical reference for endoscopic surgery ${ }^{(1-3)}$. The SPF can vary in shape and size, suggesting the presence of anatomical variation in the entry of arterial irrigation, i.e., in the ramification of the sphenopalatine artery. This is a relevant condition for the treat- ment of severe nasal haemorrhage, which may lead to errors in artery ligation.

On this basis, the objective of the present study was to describe the numerical variation of the SPF and its location in relation to the bone crista of the middle turbinate and to establish morphometric anatomical references by measurements that might facilitate its endonasal identification, causing arterial ligation to be more rapid and effective.

\section{METHODS}

\section{Specimens}

Anatomical studies of the nasal cavity were conducted on 54 hemiskulls and 12 hemifaces of adult white and non-white cadavers of both sexes in the Laboratory of Anatomy, Dental School of Bauru, University of São Paulo, Bauru, SP, Brazil. The hemiskulls were bone specimens obtained by median sagittal section, and the hemifaces, obtained by the same sagittal section, were from cadavers fixed in $10 \%$ formol. The speci- 
mens were rigorously selected and were in an excellent state of conservation, guaranteeing the reliability of the result.

The study consisted of three stages.

\section{Visual observations}

The objective of the first stage was to identify the SPF and its numerical variation by visual observation of the hemiskulls followed by photographic documentation.

\section{Anatomical observations}

In the second stage, anatomical observations were carried out to localize the SPF in relation to the adjacent structures situated in the nasal cavity, i.e., by identifying the position of the bone crista of the middle turbinate (MNT) in relation to the foramen. The following criteria were adopted: the SPF would be considered to be located in the superior nasal meatus when the posterior extremity of the bone crista of the MNT pointed in the direction of the anterior and inferior margin of the SPF; in the middle nasal meatus when the bone crista of the MNT pointed in the direction of the anterior and superior margin of the SPF; between the middle and superior nasal meatuses when the bone crista of the MNT pointed in the direction of the midline of the SPF. The specimens that presented the bone crista of the superior nasal turbinate were also identified in relation to the SPF.

\section{Anatomical measurements}

The third stage consisted of the determination of the anatomical references for the localization of the SPF by measurements of its distance, and at which angle, from the most concave point situated below the anterior nasal spine (bANS) in the 54 hemiskulls. Next, to obtain more reliable and precise parameters because of their dimensions closer to those of living tissues, measurements were made in 12 dissected hemifaces. The dissections were made under a DF Vasconcelos light microscope with a $12.5 \mathrm{~mm}$ eyepiece and a $200 \mathrm{~mm}$ objective. The endonasal route was used for access to the SPF, the mucosa was removed from the posterior lateral wall of the nasal cavity from the floor to the exposure of the foramen and of the entire bony part corresponding to its inferior and anterior margin. After dissection of this last structure, the branches of the sphenopalatine artery were visualized and documented photographically. The computer program Image Tool, version 3.0, elaborated by the group of Dr. Don Wilcox, University of Texas Health Science Center in Santo Antonio (UTHSCSA) and commercialized by the Install Shield Corporation SA, was used to make measurements on the photographs of the hemiskulls and dissected hemifaces. A line corresponding to the distance between the anteroinferior margin of the SPF close to the ethmoid crista and the most concave point located below the anterior nasal spine, denoted bANS, was traced virtually on each photograph with the Image tool 3.0 (Figure 1). This distance, in mm, was denoted bANS-SPF. Next, a line

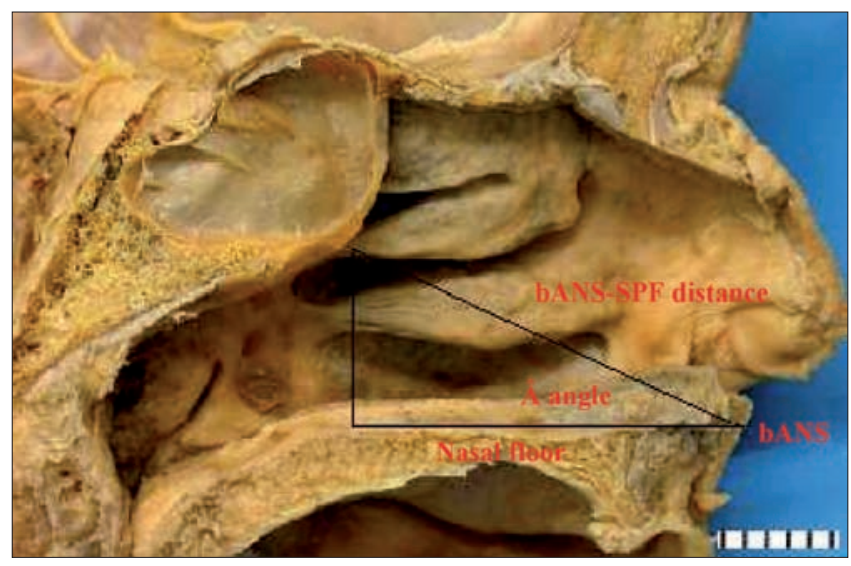

Figure 1. Left lateral wall of the nasal cavity in a hemiskull: schematic presentation of the morphometric studies. The following measurements were made: bANS-SPF distance - distance between the lower margin of the sphenopalatine foramen and the point below the anterior nasal spine; $\hat{A}$ angle - angle formed by the bANS-SPF line and the nasal floor. SPF, sphenopalatine foramen; bANS, point below the anterior nasal spine.

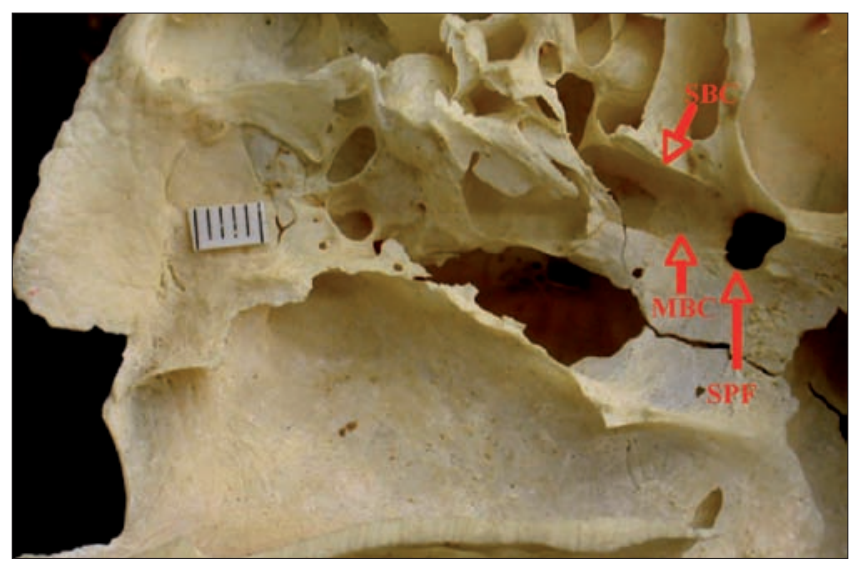

Figure 2. Single sphenopalatine foramen located in the upper nasal meatus of the right lateral bony wall of the nasal cavity. Presence of the bone crista of the superior nasal turbinate. $\mathrm{MBC}$, bone crista of the middle turbinate; $\mathrm{SBC}$, bone crista of the superior nasal turbinate; SPF, sphenopalatine foramen.

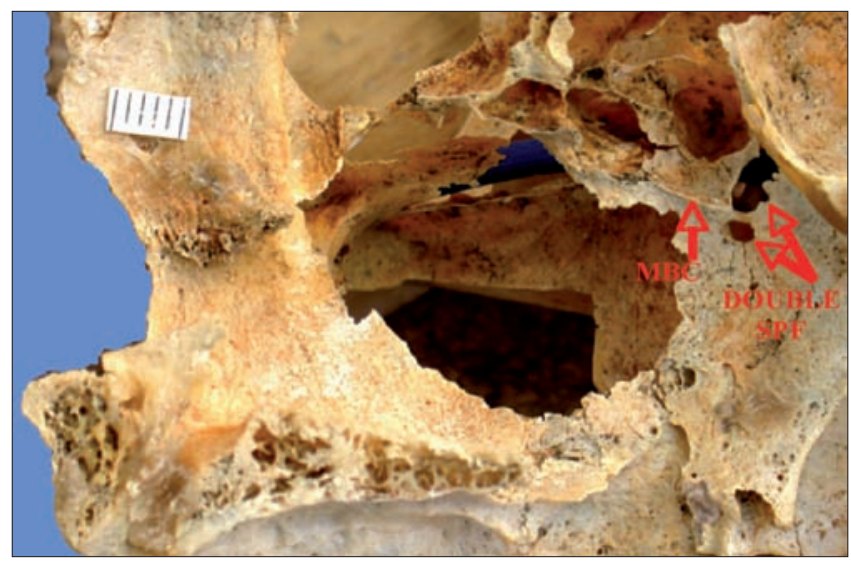

Figure 3. Double sphenopalatine foramen located in the superior and middle nasal meatuses of the right lateral wall of the nasal cavity. MBC, bone crista of the middle turbinate; SPF, sphenopalatine foramen. 
parallel to the nasal floor was similarly traced on each photograph in the anteroposterior direction, forming with the distance line (bANS-SPF) the angle denoted $\hat{A}$, expressed in degrees (Figure 1). Data are reported as means \pm SD and range for each measurement with centesimal approximation.

The photographic documentation of the bone specimens and of the dissected specimens was obtained with a Nikon Coolprix 990 digital camera. A scale equivalent to $5 \mathrm{~mm}$ was used as a dimension reference before taking each photograph (Figure 1). A total of 66 color photographs were recorded on a CD-ROM.

\section{Statistical analysis}

The mean data obtained for the dissected and bone specimens were compared by the t-test for independent samples, with the level of significance set at $\mathrm{p}<0.05$.

\section{RESULTS}

In the anatomical study of the numerical variation of the SPF in hemiskulls $(\mathrm{n}=54), 47$ specimens $(87 \%)$ were found to have a single orifice (Figure 2), while six specimens (11.1\%) had a double orifice (Figure 3) and only one had a triple orifice (1.9\%) (Figure 4). Thus, more than orifice was present in six specimens (13.0\%).

In the study regarding the location of the SPF in the nasal cavity in relation to the bone crista of the MNT, the SPF was located in the superior nasal meatus in 44 of the 54 hemiskulls (81.4\%) (Figure 2), between the middle meatus and the superior meatus in $8(14.8 \%)$ (Figures 3 and 4 ), in the middle meatus in one case, and in one case its location could not be identified.

In the four specimens with a double SPF, one orifice was on top of the other, with the superior one being smaller than the inferior one. In these specimens, the ethmoid bone crista pointed in the direction of the SPF, in such a way that it indicated that one was superior and the other inferior (Figure 3). In the other two specimens, the ethmoid bone crista pointed towards the inferior margin of the SPF, locating it in the superior nasal meatus.

The anatomical studies permitted the identification of the bone crista of the superior nasal turbinate pointing towards the $\mathrm{SPF}$ in 30 specimens (55.6\%) (Figures 2 and 4).

The study of the measurements relative to the location of the SPF demonstrated a mean bANS-SPF distance of $55.24 \pm 385$ (range: $64.95-48.25 \mathrm{~mm}$ ). The mean value of the $\hat{A}$ angle was $25.82^{\circ} \pm 5.67$ (range: 36.20 to $12.26^{\circ}$ ). The same measurements were also made in the dissected hemifaces (Figure 5), with a mean bANS-SPF distance of $58.94 \pm 4.36 \mathrm{~mm}$ (range: 68.35 $51.50 \mathrm{~mm}$ ) and a mean $\hat{A}$ angle of $26.20^{\circ} \pm 5.64$.

Comparison of the measurements made in the hemifaces and hemiskulls showed that the mean bANS-SPF distance was significantly longer in the hemifaces $(p=0.005)$ than in the

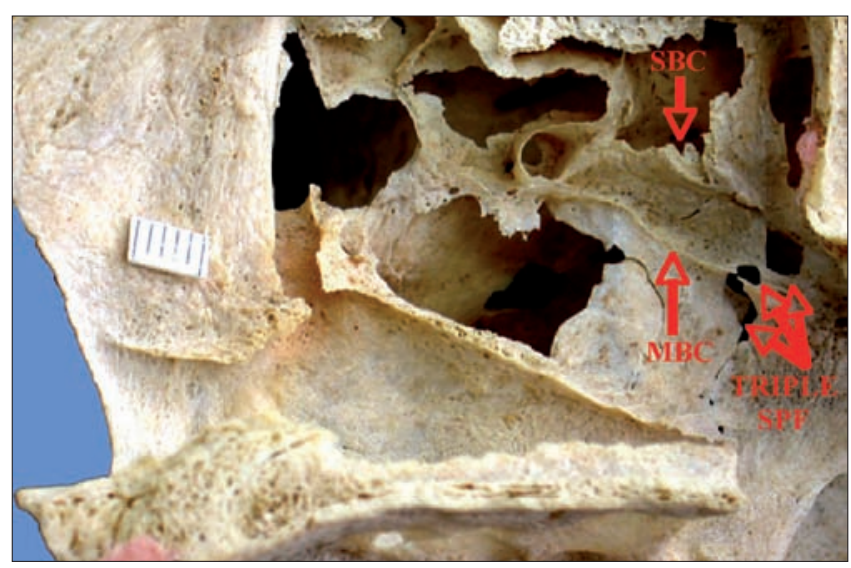

Figure 4. Triple sphenopalatine foramen located in the superior and middle meatuses of the right lateral wall of the nasal cavity. Note the presence of the bone crista of the superior nasal turbinate. SBC, superior bone crista, MBC, bone crista of the middle turbinate; SPF, sphenopalatine foramen.

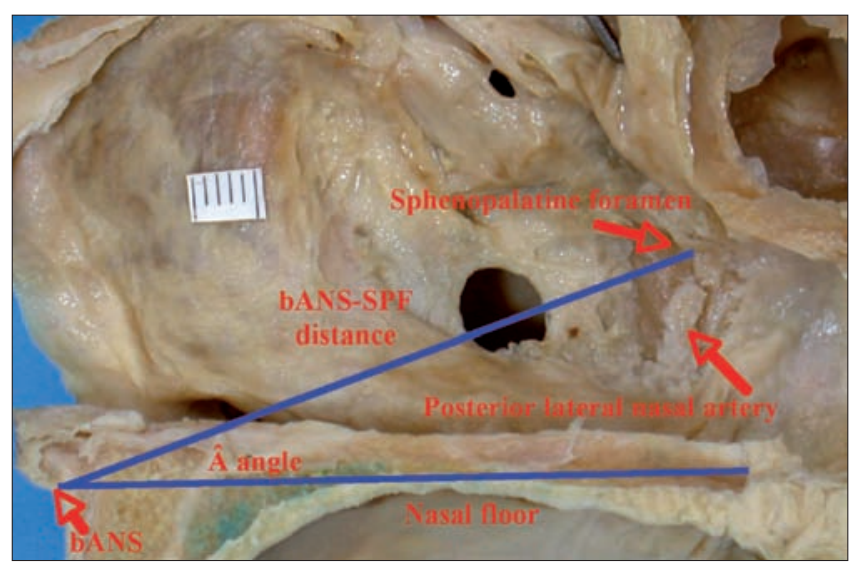

Figure 5. Right lateral nasal wall in a dissected hemiface Posterior lateral nasal artery, sphenopalatine foramen and measurements made in the nasal cavity. bANS, point below the anterior nasal spine; Â angle, angle formed by the nasal floor line and the distance between the point below the anterior nasal spine and the sphenopalatine foramen (bANS-SPF).

hemiskulls, whereas no significant difference in $\hat{A}$ angle was observed between hemiskulls and hemifaces $(p=0.15)$.

\section{DISCUSSION}

The present results corroborate literature data regarding the numerical variation of the SPF, with one to three sphenopalatine foramina being detected in the specimens studied ${ }^{(4,5)}$. Most of the specimens in our sample had a single orifice (87.0\% - 47 specimens) and the remaining ones had more than one (13.0\% - seven specimens). Our results are similar to those reported by Nikolic ${ }^{(5)}$, although the percentages differed, since Nikolic found $38.5 \%$ of specimens having more than one orifice in a sample of 840 specimens, whereas our percentage was only $13.0 \%$.

Six of the specimens with more than one orifice detected in our sample (11.1\%) had a double orifice. This frequency is sim- 
ilar to that reported by Bagatella ${ }^{(1)}(10.0 \%)$ and by Wareing and Padgham ${ }^{(3)}$ (12.0\% of 220 specimens). Only one specimen had a triple orifice in our sample (1.9\%), representing a rare finding, in contrast to Nikolic ${ }^{(5)}$ who detected 39 specimens with three orifices, corresponding to $5.5 \%$ of his large sample.

The numerical variation of the SPF may probably be the major factor explaining the surgical failures occurring when the branches of the sphenopalatine artery are ligated for the treatment of nasalhaemorrhages ${ }^{(1,3)}$. This hypothesis is based on the anatomical and numerical variation that may occur in the entry of the major branches of this artery, i.e., the septal artery and the posterior nasal artery ${ }^{(6)}$. Indeed, Lee et al. ${ }^{(7)}$ recently demonstrated the presence of two to four branches of the sphenopalatine artery before the outlet of the SPF. Thus, it may be assumed that, in anatomical-surgical terms, the numerical variation of the SPF corresponds to the variation of the branches of the sphenopalatine artery, thus representing a complicating factor in surgery. In this respect, Schwartzbauer et al. ${ }^{(8)}$ proposed that, if dissection is not performed up to the posterior portion of the SPF, surgical failure may ensue due to the presence of arterial branches exiting from accessory foramina.

In view of the importance of the identification of the ethmoid bone crista of the palatine bone as an anatomo-surgical reference for endonasal access to the location of the SPF, we observed that in 44 bone specimens $(81.5 \%)$ the distal extremity of the ethmoid bone crista was directed towards the inferior margin of the SPF, which was located in the superior nasal meatus. In eight specimens (14.0\%) the SPF was located between the superior and middle meatus, and in only one specimen $(1.9 \%)$ it was fully located in the middle meatus. These results disagree with those reported by authors who position the SPF only in the superior nasal meatus ${ }^{(2,7)}$, but corroborate those reported by Bagatella ${ }^{(1)}$ who observed that $85 \%$ of the SPF are located in the superior nasal meatus, 5.0\% in the middle nasal meatus, and $10 \%$ between the two meatuses. Wareing and Padgham ${ }^{(3)}$ also reported location of the SPF in the superior nasal meatus in $35.0 \%$ of cases, and between the superior and middle meatuses in 56.0\% and 9.0\%. It should be pointed out that this location is variable in bone skulls. However, for the treatment of severe nasal haemorrhage, during the ligation of the branches of the sphenopalatine artery a mucoperiosteal flap is made above and below the middle turbinate at about $1 \mathrm{~cm}$ from its tail, i.e., in the middle nasal meatus, to avoid surgical failure by not detecting the $\operatorname{SPF}^{(9)}$.

In the third stage of our study, we made measurements aiming at detecting the location of the SPF. The first consisted of measuring the distance from a point below the anterior nasal spine and the SPF (bANS-SPF), which yielded a mean value of $55.24 \pm 3.85 \mathrm{~mm}$. We also measured the angle formed by the distance line and the line corresponding to the anterior nasal floor, whose mean value was $23.52^{\circ} \pm 5.67$. Since we did not find references in the literature regarding these measurements, any comparison with other studies is inviable and it is our understanding that we can state that the data presented here are original and have not been published previously.

The literature report whose methodology was closest to the one used in the present study was that by Lee et al. ${ }^{(7)}$ who recorded a result of $49.1 \pm 3.7 \mathrm{~mm}$ for the nasal limen-SPF distance and a result of $59.4 \pm 4.2 \mathrm{~mm}$ for the nasal opening-SPF distance. In our study, the mean bANS-SPF distance for the hemifaces was $58.98 \pm 4.36 \mathrm{~mm}$. Our measurements are close to those obtained by the Lee's group; however, despite the similarity to the most anterior point measured by this group, it is not possible to compare measurements since the measuring methods and anatomical references used were different. Analysis of our data suggests that a distance of 54 to $63 \mathrm{~mm}$ may serve as reference in endonasal surgeries for severe nasal haemorrhage, although these values still need future confirmation in studies of surgical practice.

The literature also indicates another modality for a more precise localization of the do SPF based on the determination of the angle in relation to the sagittal plane of the head. Within this context, Portman et al. ${ }^{(10)}$ advised that the foramen should be encountered at an angulation of 15 a $20^{\circ}$ with the plane in question when using the light microscope for endonasal surgery. During the same decade, Bagatella ${ }^{(1)}$ suggested an angulation of zero to 30 degrees in relation to the same plane for these surgical procedures. The measurements obtained here were $26.20 \pm 5.64^{\circ}$ and $23.52 \pm 5.67^{\circ}$ for the hemifaces and bone specimens, respectively. Because of these results, our measurements indicate the occurrence of $20-32^{\circ}$ variation of the angle for hemifaces and of $18-29^{\circ}$ for the bone specimens. Since the hemiface material most resembles the in vivo human material, we propose that this would be the minimum angulation necessary for the insertion of the endoscope during endonasal surgeries for ligation or cauterization of the branches of the sphenopalatine artery.

The distance was significantly greater in the hemifaces a feature explained by that, in cadavers, the entire mucosal connective tissue lining is present on the skeleton, thus generating the volumetric variation observed in the present study. If we project these data to in vivo situations, we can predict a greater variation due to the presence of the system of vasodilation and constriction of the nasal turbinates and we may estimate that, in the presence of hypertrophy, the SPF may reach a more anterior position corresponding to up to one quarter of the tail of the middle turbinate ${ }^{(6)}$.

In view of the gaps existing in the literature regarding more precise anatomical references for the localization of the SPF, it is possible to appreciate the value of the measurements made 
in the present study, i.e., bANS-SPF distance and $\hat{A}$ angle. Together with the visual information, these measurements will permit a more precise access to the orifice during endonasal surgeries, reducing the risk of intraoperative complications and the surgical time, in addition to being of heuristic value for the training of students in this area.

Mapping the position of the SPF on the lateral nasal wall provided a better understanding of its anatomy, and consequently provided the desired foundation for the improvement of microendoscopic techniques. Specifically, the present study contributed to the surgical treatment of severe nasal haemorrhage, assuring better safety to the surgeon.

\section{CONCLUSIONS}

The present study demonstrated that in most of the specimens studied the STF was single and was located in the superior nasal meatus, although double and triple SPF were also found. Based on the mean distances obtained in the present study $\left(55.24 \pm 3.85\right.$ and $58.94 \pm 4.36 \mathrm{~mm}$ for bANS-SPF and $25.82^{\circ}$ $\pm 5.67^{\circ}$ and $26.20^{\circ} \pm 5.64^{\circ}$ for the $\hat{A}$ angle for hemiskull and hemifaces, respectively), we suggest that these values represent distance references for the use of the endoscope in endonasal ligation or cauterization of the branches of the sphenopalatine artery in our population.

\section{REFERENCES}

1. Bagatella F. Vidian nerve surgery revisited. Laryngoscope 1986; 96 : 194-197.

2. Bolger WE, Borgie RC, Melder P. The role of the crista ethmoidalis in endoscopic sphenopalatine artery ligation. Am J Rhinol 1999; 3: 81-86.
3. Wareing MJ, Padgham ND. Osteologic classification of the sphenopalatine foramen. Laryngoscope 1998; 108: 125-127.

4. Lang J. Paranasal Sinuses. Clinical anatomy of the nose, nasal cavity and paranasal sinuses. New York, NY: Thieme; 1989.

5. Nikolic V. Variations du trou sphéno-palatin. Acta Anat (Basel) 1967; 68: 189-198.

6. Navarro JAC, Toledo Filho JL, Zorzetto NL. Anatomy of the maxillary artery into the pterygomaxillopalatine fossa. Anat Anz (JENA) Berlin 1982; 152: 413-433.

7. Lee HY, Kim HU, Kim SS et a1. Surgical anatomy of the sphenopalatine artery in lateral nasal wall. Laryngoscope 2002; 112(10): 1813-1818.

8. Schwartzbauer HR, Shete M, Tami T. Endoscopic anatomy of the sphenopalatine and posterior nasal arteries: implications for the endoscopic management of nasal haemorrhage. Am J Rhinol 2003; 17: 63-66.

9. Voegels RL, Thomé DC, Iturralde PPV, Butugan. Endoscopic ligature of the sphenopalatine artery for severe posterior nasal haemorrhage. Otolaryngol Head Neck Surg 1999; 13: 137-140.

10. Portmann M, Guillen M, Chabrol M. Eletrocoagulation of the vidian nerve via the nasal passage. Laryngoscope 1982; 92: 453-455.

Wilma T. Anselmo-Lima

Department of Ophthalmology, Otorhinolaryngology and Head and Neck Surgery Faculty of Medicine of Ribeirão Preto

University of São Paulo

14049-900 Ribeirão Preto, SP

Brazil

Tel: +55-16-3602-2862

E-mail: mcecilia@hcrp.fmrp.usp.br 